\title{
Towards complete knowledge for complex problems resolution
}

\section{Richard Gagnon ${ }^{A}$}

Bruno Santos Ferreira ${ }^{B}$

Gilberto Lacerda dos Santos ${ }^{c}$
Professor, Département d'études sur l'enseignement et l'apprentissage, Faculté des sciences de l'éducation, Université Laval, Québec, Canada

B Ph.D student, Département d'études sur l'enseignement et l'apprentissage, Faculté des sciences de l'éducation, Université Laval, Québec, Canada

C Professor, Departamento de Métodos e Técnicas, Faculdade de Educação, Universidade de Brasília, Brasília, Brazil

\section{Keywords}

Complete knowledge;

complex problems;

meaningful knowledge;

symbol.

\section{Article Info}

Received 20 May 2019

Received in revised form 29 July 2019

Accepted 6 August 2019

Available online 13 December 2019

DOI: https://doi.org/10.37074/jalt.2019.2.s1.3

\section{Abstract}

Human beings are complex. They learn through means of very different natures - thought, feeling, sensation, intuition — that complement each other without really understanding one another. Truly ideal knowledge would nevertheless involve all these means developed to their full potential and harmonized among them, which is almost impossible since, generally, one or two of them overwhelm the others. However, all would be necessary to understand and solve the crucial and equally complex problems - such as the ones related to immigration and climate change - that only a fully integrated multidisciplinary approach would allow dealing with adequately. It is in this perspective that we explore various categories of knowledge (meaningful, encyclopedic, etc.), as well as how and to what extent we can promote the development of what we have called "complete knowledge", i.e., the richest and most complex that is accessible to an individual or a community. This would imply in practice to engage the learner with all the learning means available to him - they are associated respectively with speculation, appreciation, sensory experience and revelation. Despite the difficulty, an opening to other points of view could then take place, from the simple but already troubling tolerance of these points of view to their gradual integration in the learner's mind. We argue that if a traditional, mostly linear, deductive approach is appropriate for the development of meaningful knowledge - provided certain characteristics of the learner, related to relevance and epistemology, are taken into account - , a dialectical approach should suit better the gradual development of the comprehensive knowledge, then increasingly best regarded as a symbol, required to foster collaborative work when multiple disciplines are involved.

N.B. Part of this article reconsiders and deepens some of the ideas presented in Gagnon and Santos Ferreira (2018, in Portuguese). The masculine gender is used solely for the sake of readability. 


\section{Introduction}

Knowledge defines the human being. According to Aristotle (2002), to look for it is a natural desire, as knowledge serves to orient every one of us with respect to the world, others and ourselves. It is inextricably embedded in almost all human activities and manifests itself in several ways and fields: mythology, philosophy, religion, art, language, science, etc. (Cassirer, 1996). This diversity of manifestations embodies all kinds of ceaseless efforts - although each time with limited success - that Humanity has made throughout History to make sense of who we are, where we are coming from, where we are going, resulting in a complex network of often incompatible elements in so many languages, vocabularies, signs, hypotheses, theories, beliefs, realities, forms, causes, purposes, etc.

Of all those ways and fields of human endeavour, Science, understood as a rational explanation of something, based on rigorous methods recognized by a specialized community, stood out in the last four or five centuries for its ever increasing repercussion in our daily lives and its quasi universal credibility. It allowed us to understand a huge amount of phenomena of all sorts, physical, biological, social, psychological, economic..., but also to develop ever more complex technological systems and devices without which our modern societies would almost instantaneously collapse. ${ }^{1}$ This merely illustrates to what extent scientific (and technical) knowledge transformed our societies and their organization. Thus, new social relations of a dynamical and complex nature have been created, particularly since the first half of the twentieth century (Bell, 1977). Similar progress with similar consequences were made in almost every field of investigation, creation and knowledge, but from their own perspective, many of them influenced by the methods and techniques developed in physical sciences since Galileo's and Newton's lifetime, but also by new ways of probing irrational and unconscious phenomena elaborated from the second half of the XIX ${ }^{\text {th }}$ century, resulting in the world that we know today.

More recently, with the advent of computers, societies began to organize themselves along the principles of communication and information processing governing these machines (Castells, 1999). One started to experience a "computerized society" organized so to speak in networks of individual societies. Consequently, the fabric of social reality is continually reconfiguring itself, our control over nature is expanding, and social relations as well as our way of perceiving the world are deeply transformed. Simultaneously, our notions of time and space "dematerialize", boosting what many authors call modernization or globalization (Tabachnick \& Koivukoski, 2004; Morin, 2014). It is no wonder then that, under these conditions, education, as we conceive it nowadays, be strongly questioned.

The fact is that until recently one could say with relative certainty and peace of mind that indeed this way of functioning worked. It succeeded in Man reaching the Moon, to stop and prevent a large number of epidemics, to feed entire populations with only a few farmers, to understand the causes and effects of many social phenomena, to connect people all over the world almost instantaneously, to fill museums and libraries with extraordinary works of art, etc., using hypotheses, controlled experiments, analyses, deductive reasoning, sophisticated technologies, trials and errors, imagination, dreaming, automatic writing, etc., meanwhile, unknowingly perhaps though with the best of intention, generating new and quite severe problems intractable by usual methods: environmental degradation, social inequalities, climate change, demographic crises, and many others, not to mention socially controversial issues opposing scientists, artists and citizens who regularly do not share the same point of view on matters of public significance (Muessig \& Aldrich, 1975).

In our contemporary world, more and more, everything intertwines, mixes up, influences everything else, breeding doubt and uncertainty that no single approach, technique or methodology, no matter how powerful, can resolve alone. New challenges require new ways of thinking and the reduction of every problem to a single point of view using an all-encompassing method is not one of them. Too many variables are involved, many of them perhaps unknown, many others probably unidentifiable as such. In other words, complexity (Morin, 2014) is among us! As a consequence, monodisciplinarity, i.e., a single way to approach something and a single perspective on it, will not suffice to accommodate our world, every discipline - scientific, social, artistic, spiritual, etc. - will have to contribute generously and openly, to the point of transcending themselves, and we shall have to innovate in order to know what to do, because we ignore it, really.

Multidisciplinarity - which consists in the study of a same question by several disciplines - and interdisciplinarity which involves the transfer of methods from one discipline to another in a collaborative spirit (Darbellay, 2011) — could be first steps as these approaches are broader and extend beyond the framework of a single discipline. However, they would end up short as the contribution of each discipline would remain within its own boundaries, juxtaposed to the others more or less like a patchwork instead of forming together a truly integrated response to the actual question, limiting a true and shared understanding of its meaning and consequences. For Darbellay (2011), the disciplinary organization of knowledge hinders and even precludes the elaboration of a unified theory or worldview articulating all or at least parts of these disciplinary contributions. This is because specialization in particular fields, hermetic from other perspectives, delineates rigid limits of understanding in these fields, creates its own vocabulary and develops techniques and theories to solve its specific problems (Gusdolf, 1977). What one needs rather is a way to transcend these boundaries separating the fields in order to reach a synthesis necessarily of a symbolic nature required to better deal with complexity, such an elusive concept. Only then could one thus accept as simultaneously valid numerous interpretations from a number of perspectives even when they appear to contradict each other, in a true transdisciplinary manner (Nicolescu, 2008).

\footnotetext{
${ }^{1}$ More precisely, science and technology are inseparable, the evolution of one triggering the evolution of the other
} 
Morin (2014) argues that complexity calls for a new approach at the epistemological, methodological and theoretical levels, as well as for the necessity to rethink the idea of fragmented and compartmentalized knowledge that dictates the logic of science production and the limits of scientific knowledge. Nicolescu (2008) adds that the classical scientific ideal is discordant with the objective and the design of a complex new knowledge. We add that rational and irrational perspectives must be reconciled, i.e. science and art, myth and science, spirituality and earthly life, and the like, in order to glimpse into true complexity.

What does this tell us from an education point of view, when we are trying to determine what we should focus our attention on in educating, preparing rather, individuals and communities to cope with the above, to enter complexity?

Indeed, the general path is rather clear, but the detailed one is almost invisible. One must first recognize that the human mind resembles the world it appears to inhabit: complex. And that this complexity can bridge the learner and the object of learning. Human being learns through means of very different natures - thought, feeling, sensation, intuition (Jung, 1971) — that complement each other without really understand one another. Therefore, truly ideal knowledge would involve all these means developed to their full potential and harmonized among them. This would generate a rich, complex and ideally balanced knowledge from a human point of view. However, this is impossible since, generally, one or two of these means overwhelm the others, particularly when we are young. With maturity and experience, given the chance and the opportunity, we may develop other means but only up to a certain extent (Jung, 1971).

It is in this perspective that we explore in this article various categories of knowledge, as well as how and to what extent we can promote the development of what we have called Complete Knowledge, i.e., the richest and most complex that is accessible to an individual or a community. We obviously do not reach definitive conclusions but we propose lines along which one could travel in order to approach this complete knowledge that we are seeking for as much as possible.

\section{A meaningful knowledge}

What is knowledge? The etymology of the term refers to its origin in the Latin word cognoscere, meaning "act or effect of knowing". Thus stated, the notion of knowledge seems simple and obvious. Inside us, we feel it as something given, intimate, familiar. But as soon as we try to understand better its true meaning, it eludes us, paradoxically transforming itself into something unknown and complex (Morin, 1986). This uncomfortable phenomenon has fascinated philosophy from its beginning and continues to do so: on one hand, what knowledge means remains an open question, but on the other, it constitutes the most solid foundation on which all human science in every field rests. It is a "catch 22 " situation, "[i]t is as if there was no starting point, since the act of thinking about knowledge presupposes that knowledge is already there, as the support and cause of thought" (Morin \& Brunet, 2000, p. 43, our translation). Nevertheless, we shall assume that there is no knowledge without a subject in possession of this knowledge. And we shall consider that knowledge partakes of the perspective of a subject in a twofold manner. On one side, it is entirely subjective, private, singular, proper to the individual, intimately related to his personal history, preferences and particular ways of thinking and acting; on the other side, it is social, public, corresponding to what is shared with other people, common with them, objective shall we say, like culture, like language. It can also be very simple, like an elementary grammar rule whose understanding involves solely the use of thought, or quite complex, to the point of incomprehensibility, like a symbol whose different interpretations are mostly inexhaustible and involve all the learning means at our disposal in an inextricable sort of way. When, therefore, can we say that we know? And when is this knowledge really meaningful? These are tough questions to answer in a satisfactory manner but many years ago one of us (see Gagnon, 2013) proposed an operational definition of what a meaningful knowledge could be, which seems to pass the test empirically (Zourhlal, 1998). The definition goes as follows:

Knowledge is meaningful for an individual when it is relevant and valid from his point of view, i.e., in accordance with his epistemological position. In the same way, knowledge is meaningful for a community when it is relevant and valid from the point of view of the community in accordance with its epistemological position. ${ }^{2}$

When these two types of knowledge coexist and are compatible with one another in an individual, it means that what he knows and considers meaningful - specifically, the social part of it - is also recognized as such by a reference community, for instance, an engineer in a community of engineers or a citizen in his social community. The individual thus trusts what he knows and trusts himself, feels confident and autonomous, and acknowledged by the reference community to which he belongs.

In view of the above, we propose the following definitions:

- A knowledge exists for a subject from two complementary perspectives: 1) individual, subjective, unobservable from the outside; 2) social, objective (i.e., from others' point of view), observable from the outside. We shall call Individual Knowledge, knowledge considered from the first perspective and Social Knowledge, knowledge considered from the second perspective. For instance, listening to a detailed explanation of a theorem in mathematics or watching a demonstration of a particular technique in automotive mechanics from experts in these fields is witnessing Social Knowledge while the experts themselves proceed from Individual Knowledge.

\footnotetext{
2 Relevance is related to the effort required to learn something and to the effect of this new knowledge on the context of the individual or the community (Zourhlal, 1998, 2015; Gagnon, 2013). To validate a knowledge means to make sure that this knowledge is true and trustworthy (Gagnon, 2013). This is done according to the epistemological position of the individual or the community. Pepper (1970) has defined and characterized the following epistemological positions: mechanicism, formism, contextualism and organicism.
} 
- Two categories of Individual Knowledge can be established: 1) private, non-sharable absolutely (sensations for instance, which must be felt by oneself, like the taste of an orange which cannot be truly grasped otherwise) or intentionally (anything that one chooses to keep to oneself, examples abound in each of us) and, therefore, invisible in the Social Knowledge; 2) public, shared with the reference community and visible in the Social Knowledge (like the common language of the reference community). In general, individual knowledge is a mixture of private and public knowledge - for instance, we all know what snow or beach means but we all have different experiences associated with them and it is likely that, strictly speaking, pure private or public knowledge do not exist in an individual. We shall call Private Individual Knowledge the first category of Individual Knowledge and Public Individual Knowledge the second category. Let us note that what we have called Social Knowledge is larger than the Public Individual Knowledge as the former manifests not only the latter (for example, the specialized vocabulary of a reference community is found in both categories) but also the Private Individual Knowledge (for instance, personal tricks or idiosyncrasies that are not easily transferable, if ever possible, to other people and therefore restricted to this category). Thus, all Public Individual Knowledge is Social Knowledge, but not all Social Knowledge is Public Individual Knowledge. It is on this sole basis that, most of the time, we distinguish the best people in their field (in sports, for example) from less gifted ones: they know something, no matter how or why, that no one else does but clearly detects and recognizes as outstanding, if not magical.

- Making use of these definitions, we shall call Individual Meaningful Knowledge, knowledge pertaining to the individual perspective of the subject, private and public, considered relevant and valid by the individual. This is simply a rephrasing of the original definition of the meaningful knowledge of an individual given above. This knowledge will be termed perfect as it corresponds fully to what the individual requires to attribute to it value, trust and truth.

- $\quad$ Finally, we shall call Public Meaningful Knowledge the public part of the Individual Meaningful Knowledge. Recall that to be recognized by the reference community, it must be relevant from the perspective of the community and validated according to its epistemological position. It is quite generally upon this category of knowledge that curricula are based.

\section{Encyclopedic Knowledge}

Meaningfulness of knowledge is generally what is wished for both by a particular individual or a society. Obviously, scientific disciplines look for it, seeing in it an ideal of perfection, not only formally but as a means to solve problems specific to them in the best possible way. As discussed earlier, knowledge gains meaning through an epistemology which links it to other knowledge forming together an organized system whose sturdiness depends on the degree of relevance and validity of its constituents. In an individual, this results in a series of strong (neuronal) interconnections constituting a network of meaningful knowledge. A large group of individuals in the same discipline would agree on quite a lot of particular meaningful knowledge specific to the domain even without a priori sharing all of it. Together, they could map the whole network on which they agree, which would constitute essentially the totality of the public meaningful knowledge associated with the domain. Likewise, a large group of individuals in every discipline could do the same. The collection of all public meaningful knowledge associated with each discipline would then constitute the totality of the recognized meaningful knowledge of Humanity at a given time. This is what we generally called a Universal Encyclopedia.

Historically, the Encyclopédie was one of the most ambitious intellectual endeavors of all time. The project, led and edited by Diderot and d'Alembert in the Age of Enlightenment, aimed at presenting a synoptic view of all the scientific, artistic and professional knowledge of the time, to constitute, in a structured manner, a synthetic and coherent totality of the then actual human knowledge. The editors meant to present the general understanding of specific knowledge and the articulations between them, as they suggested in their Discours préliminaire (Guilbaud, 2017). The work would thus appear both as an encyclopedia, detailing the general course and evolution of human knowledge, and as a dictionary, presenting what was considered essential to know about each specific entry. The hope was that, assuming a minimal common vocabulary among the different disciplines and topics, the entries would explain one another as in a musical counterpoint.

Pursuing our work of defining different types of knowledge, we shall quite naturally call Encyclopedia, partial or universal depending on its scope, that kind of meaningful knowledge which, obviously in this case, contains nothing but public knowledge.

Reduced to the individual, we shall call Personal Encyclopedia all individual meaningful knowledge including both their public and private part. It is by definition perfect since it only contains meaningful knowledge. The term Private Personal Encyclopedia will refer to the whole of the private knowledge associated with individual meaningful knowledge, and the term Public Personal Encyclopedia, to the totality of the public knowledge associated with individual meaningful knowledge. The subset of the meaningful social knowledge of an individual ${ }^{3}$ will be called Perfect Social Personal Encyclopedia and the set of all Perfect Social Personal Encyclopedias will constitute the entire meaningful knowledge of Humanity, i.e., a true Universal Encyclopedia, the closest form of Social Complete Knowledge that what one can think of. This very inclusive definition of Perfect Social Personal Knowledge thus leads to the easily recognized fact that Humanity knows much more in a meaningful way

${ }^{3}$ It corresponds to the totality of individual meaningful knowledge, including idiosyncrasies, considered from the social perspective (see section 2). 
than what can be made objective in words or otherwise. In a sense, it can be said to contain a very precious portion of human freedom, hopefully acknowledged as such by most of us.

\section{Individual Complete Knowledge and the}

\section{World Wide Web}

Several observations can be made when we consider all the elements that have been presented above. Some are rather obvious since we are used to seeing them retained and implemented in the majority of the learning environments that we know of. Others are more problematic because they raise questions that we do not know how or do not always want to resolve.

Let us consider, first, the individual meaningful knowledge. We declared it perfect because it has all the characteristics that an individual requires to value and trust his knowledge. On the one hand, it is desired by him, since he considers it useful, interesting, pleasant to know or for some other reason which, from his point of view, confers to knowledge its relevance. On the other hand, it has been validated in the way that the individual favours when he wants to validate his most valuable knowledge, i.e., in conformity with his epistemological position or, in practice, with his learning style according to Kolb's classification (1984). Clearly, a given piece of knowledge can be singular, at the limit known by only one individual, it can also be false for others, but for this individual, it is loaded with meaning and precious. If it ever becomes necessary to alter this knowledge, it will be difficult.

Facing the individual meaningful knowledge, one finds the public, collective or social meaningful knowledge. It is shared by a community of individuals favouring the same epistemological approach, the same methods of knowledge validation, like the specialists of a domain, or the same language, the same culture, like a homogeneous society. Any observation or characteristic that could be attributed to individual meaningful knowledge would essentially also apply to public meaningful knowledge as it concerns a distinct community - thus comparable to an individual - in a society that comprises many. Seen from this angle, a public meaningful knowledge can be, paradoxically, singular, but also false or unacceptable to other people or communities.

In general, any individual living in a society possesses hybrid knowledge, i.e., made of public and private elements. For instance, all mechanical engineers master the principles of fluid mechanics or the mechanical properties of materials, they are common knowledge in this community, but the experience of each engineer about these contents is singular and partly unconscious, so that the resulting knowledge forms a composite mixture, partially indeterminate, peculiar to each. It will be recognized by society only if it has been validated by it - more precisely the public elements which is done through its educational institutions and professional orders. A first source of conflict then arises, when what is relevant for an individual is not necessarily relevant for the society or the group of individuals concerned. For instance, the history of mechanical engineering does not usually enter the curricula universities propose in the field, which could frustrate a student showing a strong interest in the matter. More damaging still, a significant disinterest in mathematics by a student will greatly affect his training in the field because engineering quite generally makes extensive use of mathematics. The same is true of the knowledge validation process. If the epistemological positions of the individual and of the reference community coincide - and the knowledge in question is relevant to both - we get back to this situation which we have described earlier as perfect, both for the individual and the reference community. Then the individual really feels at home in this community who, reciprocally, acknowledges him from the outset as one of its members. But if, otherwise, the modes of knowledge validation do not coincide, if, for example, a strictly analytical approach of the mechanistic type - which considers the whole as the sum of its parts, as it is common in mechanical engineering - is privileged on the one hand, but that on the other, an organic-type approach - which maintains that the whole is more than the sum of its parts, as biologists often proclaim - another problem appears, because the individual has no choice but to demonstrate to the reference community that he knows and shares its methods. In practice, the individual most often chooses to adapt to the reference community, at least while being evaluated, and returns to his preferences whenever possible. Anyone with an experience in teaching will recognize this phenomenon! In those situations, knowledge loses some of its perfection. A few years ago, Gagnon (2015) proposed some ways to implement gamification in education and training environments taking into account similar issues. The interested reader may refer to his analysis.

Still, perhaps surprisingly, perfect knowledge is not ideal knowledge, neither for the individual nor for the community, as it is profoundly exclusive. It retains as meaningful only what fits with them, regarding relevance and validity. This explains, in particular, why different highly educated individuals specialized in different fields might not get along, to the point of denigrating each other sometimes, despite their excellent reputation in their respective fields; or why, quite often, the various faculties of a same university are wary of one another, for poetry or theology, for example, rarely speak the language of experimental sciences. This reflects the idea that the perfect personal or public encyclopedias, whether they deal with science, literature, politics, philosophy, technology or religion, complement each other, exposing our multiple perspectives on the world, but they do not necessarily communicate well with each other, oftentimes contradicting and denying one another. The effects can be observed daily!

To overcome this potentially explosive problem, we should, in the spirit of what Gagnon (2013) previously proposed, promote the development of what can be called Complete Knowledge, i.e., a knowledge necessarily imperfect since it can never reach its full development, but nevertheless the richest and most complex that is accessible to an individual or a community. In practice, this would imply making sure to engage in learning all the cognitive means available to human beings, namely thought, feeling, sensation and intuition, 
associated with respectively speculation, appreciation or evaluation, sensory experience, and revelation (Gagnon, 2013). Despite the difficulty - we will come back to this question later - a progressive opening to other points of view should then take place, from the simple, but already troubling, tolerance of these other points of view in the mind of the learner to their, hopefully, evermore complete integration. Fortunately, it is such an approach that we are witnessing more and more nowadays, since the concepts of pluri-, multi-, inter-, even transdisciplinarity become increasingly relevant in our discourse and practice. However, we are still at the very early stages of the process. The fact is that we do not really want such progress to be made, that quite the contrary we fight it vigorously, because it implies to assume and accept in ourselves or within a community the coexistence of, perhaps fiercely, opposing positions, that perfect knowledge is incomplete and not the only valid one we thought it was, that the very foundations of our lives is, therefore, debatable. A process quite similar to grief is then required, and it costs a lot. For several centuries, we specialized, separating human activity into increasingly sharp areas of study, investigation, creation or practice, with, as a consequence, the gradual abandonment of a general culture $^{4}$ that constituted bridges between humans of different professions and trades. Since then, each specialty continually asks for increasingly specific knowledge and longer training, relegating to oblivion contents of general culture. Under these conditions, the "single thought" finds a breeding ground of the most fertile, which accentuates the differences between the various domains to the point of fracture. A brief overview of vocational, technical or university curricula is enough to convince us of this. We shall never insist too much, these are foreign "countries" to be reconciled, "countries" in which we speak, the one, the language of the spirit, the other, the language of the heart, the other still, the language of the body, when not, as in the arts of creation and the spiritual domains, the incomprehensible language of intuition. A real Tower of Babel! Polyglotism is not innate! Nevertheless, it is by cultivating the less familiar aspects of ourselves, those that we reject as negative or improper, that gradually we can achieve the necessary opening to other points of view. As an example, it is by admitting that theoretical knowledge can find practical applications that the most obstinate theoreticians of physics, who a priori do not care for applied science, can understand and accept more generously the point of view of engineers; and by admitting as valid, even without understanding them, the economic arguments of administrators, business people or accountants, that the most stubborn defenders of the environment, of education or health care will contribute to the solution rather than the aggravation of the problems by their stubbornness. Paradoxically, if this is so, the ideal knowledge remains and will remain forever partially developed, imperfect, a source of tension necessary to its vitality, but also a source of serenity in front of our undeniable impotence towards the impossible. Complete Knowledge, however, comes with a price: the painful sacrifice of a perfect knowledge, fully meaningful, the utopia par excellence of the scientific activity.

One will easily concede that education and training oriented this way will not be popular, that specialized curricula are much preferred instead, as they are much simpler to carry out and much more profitable in the short term, but that they will likely be more harmful in the long run, from the perspective of sustainable development in particular, although this is questionable.

But all things considered, would not the Internet provide a plausible model pointing towards Complete Knowledge, at least on the public, social and collective side? One may certainly recognize in it a rather faithful image of human knowledge and the various mind dispositions and attitudes underlying it. In particular, one finds in the Internet a great deal of meaningful knowledge relevant and valid for communities of individuals rightfully recognized socially in almost every area of human activity. One encounters sites of professional associations, corporations, for-profit or not-forprofit organizations, private and public institutions of all kinds, ministries or governments, developed by or in collaboration with authoritative experts, that we can usually trust. All these sites would form the essence of a perfect social encyclopedia growing and improving day by day and exceeding by far in quantity, accessibility and possibilities of use all classical encyclopedias, compendiums, dictionaries and works of all kinds traditionally published on paper, which are now found almost entirely on the Web of which they constitute a very small part. Because of their diversity, these sites also cover a set of knowledge fields extremely representative of human activity as well as the epistemological positions and methods of knowledge validation that prevail in these fields. In this respect, it can be compared with a traditional university where the deeply original cultures of its faculties generate almost spontaneously affinities and oppositions. This is why many other sites intent to counterbalance these "perfect" sites, as their authors defend other views. This is obviously healthy and desirable when it is done with respect and dignity, but it exacerbates the tensions and antagonizes the parties otherwise. All these sites could be put to use advantageously in learning environments, either for the purpose of specialization, for those seeking the development of meaningful knowledge in a given field, or to question status quo in a reference community. In the latter case, it would allow to acquire a different kind of knowledge, strange or weird perhaps as some unusual clothes, but enriching previous knowledge and conferring on the result an imperfect character that tempers and nuances early certainties and weakens dogmatisms. However, learners in these environments should be taught to balance things down to reduce cynicism, inappropriate behaviour, indoctrination, manipulation and other malfeasance of which the human being is obviously capable, particularly on the Internet, where everything, by far, is not worth the same.

Moreover, alongside the knowledge that can be considered socially meaningful on the Internet is an undoubtedly larger quantity of non-meaningful knowledge; most likely because they have not been validated socially. They fall within the opinion, which does not satisfy a "well-made-head". People in education are well aware of the problems that this creates, but they are a far cry from resolving them. ${ }^{5}$

\footnotetext{
${ }^{4}$ For the lucky few fortunate enough to attend school, the vast majority having been confined to ignorance.

${ }^{5}$ Will they ever be solved considering how fast the Internet is changing?
} 
Individuals also find meaningful knowledge on the Internet, but which a community could not sanction. It is about singular knowledge, personal or specific to few people, essentially private. Limited in scope, this knowledge is anecdote to other people, although it may interest some in the manner of a work of fiction or a biography and possibly give rise to generalizations. Social networks are full of this type of knowledge that we should preferably call information. Of course, caution is here again necessary to avoid confusing the particular with the general.

\section{Discussion and conclusion}

Human beings are complex. The learning means at their disposal are the same for everyone, but they are not equally developed, neither in each person nor from one individual to another. They complement each other but one could say that they all speak different languages. As a result, a truly ideal knowledge, i.e., one that would fully manifest the contribution of all these learning means developed to their full potential and harmonized with each other, is not possible and we must be satisfied with a lesser deal. Harmonization is inevitably difficult, painful too, and always partial since one or two of these learning means take precedence over the others. These strongly contribute to determine our fields of interest, our most basic epistemological position and our dominant learning style, therefore our perfect knowledge from our perspective, which altogether constitutes our perfect personal encyclopedia. If we add to it everything else that we know, that do not satisfy all the conditions for meaningfulness, and is often unconscious, we get the whole of our knowledge, our complete knowledge.

From another point of view, the richness of what we know about a given object of knowledge depends on the contribution of those learning means that we generally neglect, the least skilled and the least valued to be clear, thus generating elements of knowledge on this object little or not meaningful and, by definition, imperfect. The resulting knowledge, mobile and evolving, will thus be, in part, meaningful and perfect, and, in part, little or not meaningful and imperfect, but it will surely be superior, more complete and preferable to the sole meaningful component. More nuanced and less affirmative, it will open in the learner perspectives that he previously ignored or depreciated, fostering acceptance and understanding of people who rely primarily on these learning means and of points of view based on them. The totality of this enriched knowledge constitutes our Complete Personal Encyclopedia.

We also live in society. It is therefore necessary that our knowledge be recognized as meaningful by it if we want to belong and feel accepted. For this to occur, individual and social interests must be compatible - they obviously may differ - , so that knowledge relevant to an individual will also be relevant to society or, more generally, to the reference community. Moreover, the knowledge validation modes accepted in the reference community will have to be mastered by the individual sufficiently to satisfy the minimal requirements of the community in this respect, even if, for him perhaps, they are less meaningful. If these conditions are met, the integration and recognition of the individual in the reference community is almost assured.

Under these conditions, several types of education and training environments of different degrees of complexity can be conceived of, depending on the goals aimed at. For instance, to teach to the learner knowledge that the reference community wishes him to master, i.e., meaningful to this community, the process remains relatively simple, since it suffices to select the relevant knowledge, to specify the objectives pursued and the knowledge validation modes accepted by the community. This type of teaching, however, will not produce, other than by chance, knowledge meaningful to the learner, but it can meet a collective need, provided one accepts the risk to sabotage the efforts of at least some learners, ill at ease with the teaching methods, even, in the worst cases, endangering them. Nevertheless, this type of device is clearly the most common choice for workplace training. But if we rather choose to generate knowledge meaningful both to the learner and to the reference community, the size of what needs to be developed will increase considerably, but not much the degree of difficulty nor the complexity attached to it. It will obviously require more work, resources and investment, but it would essentially consist of an appropriate juxtaposition of a number of learning environments of the first type, ${ }^{6}$ each one embodying a single epistemological position or a single learning style. However, a new difficulty arises since the degree of relevance of the knowledge looked for and the actual reasons why it becomes relevant to a particular individual may vary with each of them. They are not predetermined as in the previous case. To help to resolve this difficulty, it should be necessary to identify, through appropriate tests, the epistemological positions, or the learning styles, of the learners in order to propose to them suitable learning activities. Bigger and more open than learning environments of the first type, this second type would appeal to those people and organizations who seek to train autonomous and confident people, equally at ease socially and well adapted to their own environment.

So far, we sought the development of perfect knowledge, limited to a reference community in the first case, both for individuals and a reference community in the second. But would it be possible, in spite of the foreseeable difficulty, to develop a third type of learning environment, one that would allow and deliberately seek the development of complete knowledge, i.e., a meaningful knowledge enriched by the contributions of the learning means usually neglected and belittled by the individual or the reference community and most often dismissed for the same reasons, painfully harmonized with the prominent learning means, and whose development requires, for all intents and purposes, the work of a lifetime? As this question is stated, a readily answer would obviously be a loud and clear "No!", but let us refine it.

Let us first remember that knowledge becomes meaningful by acquiring relevance and validity. To become relevant, it

\footnotetext{
${ }^{6}$ Normally five, i.e., four to account for the four fundamental epistemological positions, or the four learning styles according to Kolb's classification, and a fifth accounting for the specific characteristics of the reference community.
} 
must be desirable to the learner, promising him, in a way, that it will bring him something good, that will have a favorable effect in his life. Otherwise, the learner will not try hard enough - if, of course, the effort required is not overwhelming him - most often because he feels that the knowledge in question is not worth it. Regarding validity, the learner must ensure that the knowledge appears true to him, convincingly, that he is not mistaken. To achieve this, he will follow an epistemological process that he trusts, which led him to constitute probably the major part of the knowledge he relies on the most, an epistemological process that mirrors his temperament and is closely related to the learning means which he privileges and which he particularly cultivated. He will have developed, consciously or not, a kind of algorithm that he scrupulously respects, so to speak, when he really wants to learn something. In this way, he increases qualitatively and quantitatively his meaningful knowledge in a more or less regular progression, connecting it from the same perspective, in a sort of linear and traditional fashion, the disciplinary perspective.

But when it comes to concede value to elements of knowledge or points of view of all kinds (intellectual, emotional, psychomotor) that the learner spontaneously considers as secondary or inappropriate, even "false", because they escape his understanding, it goes otherwise, as these elements and points of view are neither desired by him, nor can they be validated in his usual way. It is this kind of feeling that we experience when a position on a given subject is defended with conviction by someone else who sees the world in a way elusive to us, like - put in a cartoonish manner - a poet in front of a scientist, or an athlete in front of an intellectual. These are, in appearance, irreconcilable representations of the world, but equally true and, therefore, complementary. However, in this case, contrary to what we concluded regarding meaningful knowledge, one cannot move from one representation to another "linearly" because they are of different natures and cannot be apprehended with the same learning means. To open to these, weird to us, representations, we must instead use the learning means that we do not master and often depreciate. It must be done step by step, avoiding direct confrontation with the opposite point of view, which is associated with the least developed of our learning means and remains unacceptable to us. Priority should be given to the learning means following in importance our most valued ones, in order to cushion the shock. For example, going from feeling to thought to intuition, in the case of a learner who favours sensation, next feeling. ${ }^{7}$ In other words, following a dialectical path, but in a minor mode. In this way, knowledge will get enriched with new and sometimes contradictory aspects and elements - that will have to be reconciled - increasingly assuming the form and characteristics of a symbol. Meanwhile, frontiers between distinct disciplines will fade out, allowing transdisciplinarity to emerge gradually as well as a positive but careful appreciation of complexity. Clearly, much work remains to be done before we meet with some of the new well-made heads.

\section{References}

Aristotle. (2002). Metaphysics. Translated by Sachs, Joe (2nd ed.). Santa Fe, NM: Green Lion Press.

Bell, D. (1977). O advento da sociedade pós-industrial: Uma tentativa de previsão social. São Paulo, Brazil: Cultrix.

Cassirer, E. (1996). The philosophy of symbolic forms. New Haven, CT: Yale University Press.

Castells, M. (1999). A sociedade em rede. São Paulo, Brazil: Paz e Terra.

Darbellay, F. (2011). Vers une théorie de l'interdisciplinarité? Entre unité et diversité. Nouvelles perspectives en sciences sociales, 7(1), 65-87. https://doi.org/10.7202/1007082ar.

Gagnon, R. (2013). Éduquer après Carl Gustav Jung suivi de métaphores et autres vérités. Québec, Canada: Presses de I'Université Laval.

Gagnon, R. (2015). Jogar para ter sentido. In A. Zouhrlal, S. B. Ferreira, C. Ferreira et al. (Eds.), Gamificação: Como estratégia educativa (pp. 76-106). Brasília, Brazil: Link Comunicação e Design.

Gagnon, R., \& Santos Ferreira, B. (2018). Tecnologia, Educação e o Conhecimento em Ambientes de Formação on-line. In C. Goulart \& G. Lacerda Santos (Eds.), Tecnologias e comunicação pedagógica (pp. 193-212). Brasília, Brazil: Viva Editora.

Guilbaud, A. (2017). L'ENCCRE, édition numérique collaborative et critique de L'Encyclopédie, Recherches sur Diderot et sur l'Encyclopédie, 52, 5-22.

Gusdorf, G. (1977). Past, present and future in interdisciplinary research. International Social Science Journal, 29(4), 580-600.

Jung, C. G. (2014). Psychological types. Princeton, NJ: University Press.

Kolb, D. A. (1984). Experiential learning: Experience as the source of learning and development. Englewood Cliffs, NJ: Prentice-Hall Inc.

Morin, E. (1986). La connaissance de la connaissance. Paris, France: Seuil.

Morin, E. (2014). Introduction à la pensée complexe. Paris, France: Points.

Morin, L., \& Brunet, L. (2000). Philosophie de l'éducation. Québec, Canada: Presses de I'Université Laval.

Muessig, R. H., \& Aldrich, J. L. (1975). Controversial issues in the social studies. A contemporary perspective. Washington, DC: National Council for the Social Studies.

${ }^{7}$ According to Jung's model of the human psyche. In this model, sensation opposes intuition and thought opposes feeling (see Jung, 2014). 
Nicolescu, B. (2008). Transdisciplinarity. Theory and practice. Cresskill, NJ: Hampton Press.

Tabachnick, D., \& Koivukoski, T. (2004). Globalization, technology and philosophy. Albany, NY: State University of New York Press.

Pepper, S. C. (1970). World hypotheses: A study in evidence. Berkeley and Los Angeles, CA: University of California Press.

Zourhlal, A. (1998). Contribution théorique et empirique à l'étude de la connaissance signifiante en contexte de formation professionnelle. Doctoral dissertation. Université Laval, Québec, Canada. Retrieved from http://ariane.ulaval. $\mathrm{ca} / \mathrm{cgi}$-bin/recherche.cgi?qu =01-0521881

Zourhlal, A. (2015). Conhecimentos e aprendizagens significativos: Algumas pistas de pesquisa para os jogos educativos. In A. Zouhrlal, S. B. Ferreira, C. Ferreira et al., Gamificação: Como estratégia educativa (pp. 126-146). Brasília, Brazil: Link Comunicação e Design.

Copyright: @ 2020 Richard Gagnon, Bruno Santos Ferreira, and Gilberto Lacerda dos Santos. This is an open-access article distributed under the terms of the Creative Commons Attribution License (CC BY). The use, distribution or reproduction in other forums is permitted, provided the original author(s) and the copyright owner(s) are credited and that the original publication in this journal is cited, in accordance with accepted academic practice. No use, distribution or reproduction is permitted which does not comply with these terms. 